\title{
OlympiAD trial: moving to a next level of treatment for patients with BRCA mutation and her2-negative metastatic breast cancer
}

\author{
Alejandro Martin Sanchez ${ }^{1}$, Armando Orlandi ${ }^{2}$, Gianluca Franceschinii ${ }^{1,3}$, Flavia De Lauretis ${ }^{1}$, \\ Daniela Terribile ${ }^{1,3}$, Antonio Franco ${ }^{1}$, Riccardo Masetti ${ }^{1,3}$ \\ ${ }^{1}$ Multidisciplinary Breast Center, Dipartimento Scienze della Salute della donna e del Bambino e di Sanità Pubblica, ${ }^{2}$ Division of Medical Oncology, \\ Fondazione Policlinico Universitario A. Gemelli IRCCS, Rome, Italy; ${ }^{3}$ Istituto di Semeiotica Chirurgica - Università Cattolica del Sacro Cuore, \\ Rome, Italy \\ Correspondence to: Alejandro Martin Sanchez, MD. Multidisciplinary Breast Center, Dipartimento Scienze della Salute della donna e del Bambino e di \\ Sanità Pubblica, Fondazione Policlinico Universitario A. Gemelli IRCCS, Largo Agostino Gemelli 8, 00168 Rome, Italy. \\ Email: martin.sanchez@hotmail.it; martin.sanchez@policlinicogemelli.it. \\ Provenance and Peer Review: This article was commissioned by the Editorial Office, Annals of Palliative Medicine. The article did not undergo external \\ peer review. \\ Comment on: Robson M, Ruddy KJ, Im SA, et al. Patient-reported outcomes in patients with a germline BRCA mutation and HER2-negative \\ metastatic breast cancer receiving olaparib versus chemotherapy in the OlympiAD trial. Eur J Cancer 2019;120:20-30.
}

Submitted Dec 30, 2019. Accepted for publication Jan 16, 2020.

doi: 10.21037/apm.2020.01.14

View this article at: http://dx.doi.org/10.21037/apm.2020.01.14

During the last 20 years, due to a widespread use of mammographic screening programs we witnessed a significant proliferation of early stage breast cancers (BCs) diagnosis, such as T1 lymph node-negative (1-4) that today represent the most frequently diagnosed invasive BCs in developed countries.

Nevertheless, up to $8 \%$ and $4 \%$ in American and European countries are still diagnosed in locally advanced or metastatic stage (5), which constitute a challenging field in modern oncology.

Metastatic patients are still struggling with median overall survival of 2-3 years and 5 -year survival of $<25 \%$ (6) whereas patients with metastatic, triple-negative breast cancer are facing a more serious challenge of median overall survival of $<1$ year (7).

Several improvements have been made, especially in BRCA germline mutation-metastatic setting, as reported in phase II studies with olaparib, an oral poly (ADPribose) polymerase inhibitor (PARPi) in combination with anthracycline and taxane regimens, that achieved a significant improvement in overall response and progression free survival (PFS) rates (8-10).

The first randomized, open- label, phase III trial comparing olaparib monotherapy to standard chemotherapy in patients with HER2-negative metastatic breast cancer carrying BRCA1/2 germline mutations was published by Robson and colleagues (OlympiAD trial), that reported promising results such as longer PFS and no significant difference in overall survival rates (11).

This milestone changed our clinical practice, transforming the triple negative breast cancer BRCA positive into a breast cancer subtype susceptible to a targeted therapy and as a consequence of these conclusions, olaparib was the first FDA approval PARPi for the treatment of patients with suspected deleterious or deleterious germline BRCA1/2 mutation, and HER2-negative metastatic breast cancer who have been treated with chemotherapy either in the neoadjuvant, adjuvant, or metastatic setting.

But furthermore than a modest absolute advantage in PFS (of about 2.8 months) current trials must ensure to this subtype of physical and mentally compromised patients a substantial improvement in their quality of life (QoL).

Therefore, the authors reprocessed their data and recently reported the impact of olaparib compared to chemotherapy on health-related quality of life (HRQoL) (11).

Despite an important rate of unavailable information, that may have biased the results of the assessments (questionnaire compliance and completion rates were lower 
in the chemotherapy treatment of physician's choice arm) the available data clearly showed an advantage in HRQoL in patients treated with PARPi versus chemotherapy, besides for nausea and vomiting scores that were better in the latter group.

This reported point against olaparib must be analyzed in a bigger picture and faced in the context of multidisciplinary breast cancer units, where integrative and alternative therapeutic perspectives can make the difference, decreasing nausea and vomiting rates $(12,13)$.

In conclusion, Robson's data showed that an innovative treatment with Olaparib, besides an improvement in PFS, in addition to intrinsically reduction of patients' hospitalization (oral therapy versus intravenous treatment) guarantees an improvement of QoL compared with chemotherapy.

\section{Acknowledgments}

Funding: None.

\section{Footnote}

Conflicts of Interest: The authors have no conflicts of interest to declare.

Ethical Statement: The authors are accountable for all aspects of the work in ensuring that questions related to the accuracy or integrity of any part of the work are appropriately investigated and resolved.

Open Access Statement: This is an Open Access article distributed in accordance with the Creative Commons Attribution-NonCommercial-NoDerivs 4.0 International License (CC BY-NC-ND 4.0), which permits the noncommercial replication and distribution of the article with the strict proviso that no changes or edits are made and the original work is properly cited (including links to both the formal publication through the relevant DOI and the license). See: https://creativecommons.org/licenses/by-nc-nd/4.0/.

\section{References}

1. Schootman M, Jeffe D, Reschke A, et al. The full potential of breast cancer screening use to reduce mortality has not yet been realized in the United States. Breast Cancer Res Treat 2004;85:219-22.

2. Kennedy T, Stewart AK, Bilimoria KY, et al. Treatment trends and factors associated with survival in T1aN0 and T1bN0 breast cancer patients. Ann Surg Oncol
2007;14:2918-27.

3. Luke C, Nguyen AM, Priest K, et al. Female breast cancers are getting smaller, but socio-demographic differences remain. Aust N Z J Public Health 2004;28:312-6.

4. Bland KI, Menck HR, Scott-Conner CE, et al. The National Cancer Data Base 10-year survey of breast carcinoma treatment at hospitals in the United States. Cancer 1998;83:1262-73.

5. Allemani C, Sant M, Weir HK, et al. Breast cancer survival in the US and Europe: a CONCORD high-resolution study. Int J Cancer 2013;132:1170-81.

6. Cardoso F, Costa A, Senkus E, et al. 3rd ESO-ESMO international consensus guidelines for Advanced Breast Cancer (ABC 3). Breast 2017;31:244-59.

7. Khosravi-Shahi P, Cabezón-Gutiérrez L, CustodioCabello S. Metastatic triple negative breast cancer: Optimizing treatment options, new and emerging targeted therapies. Asia Pac J Clin Oncol 2018;14:32-9.

8. Tutt A, Robson M, Garber JE, et al. Oral poly(ADPribose) polymerase inhibitor olaparib in patients with BRCA1 or BRCA2 mutations and advanced breast cancer: a proof-of-concept trial. Lancet 2010;376:235-44.

9. Gelmon KA, Tischkowitz M, Mackay H, et al. Olaparib in patients with recurrent high-grade serous or poorly differentiated ovarian carcinoma or triple-negative breast cancer: a phase 2, multicentre, open-label, non-randomised study. Lancet Oncol 2011;12:852-61.

10. Kaufman B, Shapira-Frommer R, Schmutzler RK, et al. Olaparib monotherapy in patients with advanced cancer and a germline BRCA1/2 mutation. J Clin Oncol 2015;33:244-50.

11. Robson M, Im SA, Senkus E, et al. Olaparib for Metastatic Breast Cancer in Patients with a Germline BRCA Mutation. N Engl J Med 2017;377:523-33.

12. Magno S, Filippone A, Scaldaferri A. Evidence-based usefulness of integrative therapies in breast cancer. Transl Cancer Res 2018;7:S379-89.

13. Ling $Y$, Yang $D$, Shao $W$. Understanding vomiting from the perspective of traditional Chinese medicine. Ann Palliat Med 2012;1:143-60.

Cite this article as: Sanchez AM, Orlandi A, Franceschini G, De Lauretis F, Terribile D, Franco A, Masetti R. OlympiAD trial: moving to a next level of treatment for patients with BRCA mutation and her2-negative metastatic breast cancer. Ann Palliat Med 2020;9(2):510-511. doi: 10.21037/apm.2020.01.14 